\title{
标准化降水指标与 $Z$ 指数在我国应用的对比分析
}

\author{
袁文平 周广胜* \\ (中国科学院植物研究所植被数量生态学重点实验室 北京 100093)
}

\begin{abstract}
摘 要 利用分布于我国不同气候区的 7 个气象台站 $1951 \sim 1995$ 年的月降水资料,比较分析了标准化降水指标 (Standardized precipitation index, SPI) 和在我国已成熟应用的 $Z$ 指数。结果表明, SPI 计算简单, 资料容易获取, 而且 计算结果与 $Z$ 指数有极好的一致性。同时, 由于 SPI 是通过概率密度函数求解累积概率, 再将累积概率标准化而 得, 具有稳定的计算特性, 消除了降水的时空分布差异, 在各个区域和各个时段均能有效地反映旱涝状况, 优于在 我国广泛应用的 $Z$ 指数。此外, SPI 还可以计算不同时间尺度的指标值, 能够满足不同水资源状况分析的要求。 以北京为例，探讨了应用 4 种时间尺度的 SPI 值反映 1951 1995 年期间的旱涝事件, 发现 SPI 能准确地反映北京 45 年间的旱涝趋势, 对于旱涝灾害有着良好的预测作用, 可用于我国的水资源评估以及不同时间尺度的干旱监测。 关键词 标准化降水指标 $Z$ 指数 干旱
\end{abstract}

\section{COMPARISON BETWEEN STANDARDIZED PRECIPITATION INDEX AND $Z$-INDEX IN CHINA}

\author{
YUAN Wen-Ping and ZHOU Guang-Sheng ${ }^{*}$ \\ ( Laboratory of Quantitative Vegetation Ecology, Institute of Botany , Chinese Academy of Sciences , Beijing 100093 , China)
}

\begin{abstract}
Droughts are the world' s costliest natural disasters, causing an estimated $\$ 6-\$ 8$ billion in global damages annually and affecting more people than any other form of natural disaster. Given the consequences and pervasiveness of droughts, it is important to assess the specialized indices that are used to assess drought severity. The standardized precipitation index (SPI) has several characteristics that are an improvement over previous indices, including its simplicity and temporal flexibility that allow it to examine both short term and long term drought conditions. Computation of the SPI involves fitting a gamma probability density function to a given time series of monthly precipitation totals for a weather station. The resulting parameters are then used to find the probability of a particular precipitation event over a given time scale. This probability is then converted to the standard normal random variable SPI index value. In this article , 1-month SPI are calculated and compared with the $Z$-index , the most widely used index in China. The results demonstrated that 1month SPI calculated for 7 observational stations are greatly consistent with $Z$-index , but that the SPI rarely relates to distributing on precipitation, avoiding some of the irregularities associated with the $Z$-index. Thus , the $S P I$ is superior to the $Z$-index in its application.

We also investigated drought and flood events from 1951 - 1995 for Beijing in greater detail. By using the 24-month SPI $\left(S P I_{24}\right)$, three well-defined drought and flood events were identified from the data series. In general , the same drought and flood events were observed using the 12-month $S P I\left(S P I_{12}\right)$ as the $S P I_{24}$, although there were some interruptions where the $S P I_{12}$ values approached zero or became negative for short periods. For 3-month periods $\left(S_{3} I_{3}\right)$, the $S P I$ values frequently fell above and below zero. These results highlight the $S P I$ characteristics at different time scales. As the time scale increases from 1 to 24 months , the SPI responds more slowly to short-term precipitation variation , and the cycles of positive (wet conditions) and negative (drought) SPI values become more visible. The possibility of calculating the SPI for different time scales enhances its analysis capacity, since it allows the estimation of different antecedent conditions in the soils. Whereas the shortest scales ( 1 to 3 months) quantifies superficial soil water, which bears a direct significance for agriculture , the longest accumulation scales (12 to 24 months) indicate the state of subsoil moisture as well as other surface and subsurface water resources. The joint consideration of different SPI scales in the analysis contributed to a satisfactory explanation of risk conditions before each flood event reported. These results indicate that the SPI is an effective index for assessing drought conditions at different time scales and should be adopted for use in China.
\end{abstract}


Key words Standardized precipitation index , $Z$-index , Drought

干旱作为一种气象灾害, 长期困扰着工农业生 产。据测算, 每年因干旱造成的全球经济损失高达 60 80 亿美元, 远远超过了其它气象灾害(Wilhite， $2000)$ 。我国平均每年受旱面积约 2159.3 万公顷， 约占各种气象灾害面积的 $60 \%$,每年因旱灾损失粮 食 100 亿公斤。随着经济的迅速发展、人口增长及 由此引起的以气候变暖为标志的全球变化的发生, 干旱有进一步加重的趋势，从而必将对我国社会经 济及人民生活造成严重的影响。同时水分也是制约 陆地生态系统的关键因子。每年全球有一半以上的 陆地生态系统面临着干旱的威胁 (Felix ,1997)。

目前, 关于干旱指标已有大量的研究 (Richard \& Heim , 2002)。由于干旱的形成原因异常复杂, 影响 因素很多, 许多干旱指标都是针对具体的研究目的 而设定，所以干旱指标具有明显的地域性和不同的 时间应用尺度。正因为如此, 不同学者利用不同干 旱指标对我国干旱区的划分仍存在不同的意见，上 下相差 5\%(47.5\% 52.5\%) , 即 48 万 $\mathrm{km}^{2}$ 的国土 是属于半干旱区还是半湿润区尚不确定 (王廷禄, 1990)。根据建立途径的不同可以把干旱指标大致 归纳为两类。一类是通过研究干旱机理, 力图细致 地反映干旱涉及的各个物理过程, 如土壤水分蒸发、 地表径流和地表水分补给等, 以提高对干旱强度和 持续时间的反映精度。这类指标以 PDSI (Palmer drought severity index) (Palmer ,1965)、KBDI (Keetch byram drought index $)^{1)}$ 、WAWAHAMO (Zierl 2001) 等为 代表。另一类干旱指标则是通过气象学方法研究降 水量的统计分布规律, 以反映干旱的强度和持续时 间。第一类指标的物理机制较为明确, 但其计算繁 杂 对资料要求较高, 部分参数不能依靠实验获得, 只能靠经验估计, 从而大大降低了计算精度。而且 由于干旱与局部的气候、土壤、植被等多种因素有 关,所以时空变异也在一定程度上降低了这类指标 的适用范围。第二类指标计算简单，所需资料容易 获取, 而且由于指标不涉及具体的干旱机理, 时空适 应性较强。代表性模型是 McKee 等 (Seiler et al. , 2002 )在评估美国科罗拉多干旱状况时提出的基于 降水量的标准化降水指标 $(S P I)$ 。该指标替代了计 算烦杂的 PDSI 能够较好地反映干旱强度和持续时
间，而且具有多时间尺度应用的特性，使得用同一个 干旱指标反映不同时间尺度和不同方面的水资源状 况成为可能，因而得到广泛应用( Hayes et al. , 1999)。

本文通过比较 $S P I$ 和在我国应用较为成熟的 $Z$ 指数(鞠笑生等, 1998) , 分析了 SPI 的应用特性及其 在我国的适用性，发现 SPI 计算简单可行，在我国不 同气候区域都具有良好的计算稳定特性，优于 $Z$ 指 数，其多时间尺度应用特性可以满足不同地区、不同 应用的需求, 可以为我国的水资源评估和不同时间 尺度的干旱监测服务。

\section{1 研究方法与资料}

\section{1 研究方法}

比较 $S P I$ 与 $Z$ 指数在全国不同气候区的一致 性，在此基础上分析两者在反映干旱程度上的差异， 考察 SPI 反映旱涝状况的优越性, 为 $S P I$ 应用于我 国的水资源评估和不同时间尺度的干旱监测提供依 据。

$S P I$ 由于不同时间、不同地区降水量变化幅度 很大, 直接用降水量很难在不同时空尺度上相互比 较, 而且降水分布是一种偏态分布, 不是正态分布, 所以在降水分析中, 采用 $\Gamma$ 分布概率来描述降水量 的变化 然后再经正态标准化求得 SPI 值。

假设某一时段的降水量为 $x$, 则其 $\Gamma$ 分布的概 率密度函数为 :

$$
\begin{aligned}
& g(x)=\frac{1}{\beta^{\alpha} \Gamma(\alpha)} x^{\alpha-1} e^{-x / \beta}(x>0) \\
& \Gamma(\alpha)=\int_{0}^{\infty} x^{\alpha-1} e^{-x} d x
\end{aligned}
$$

式中, $\alpha$ 为形状参数, $\beta$ 为尺度参数, $x$ 为降水量， $\Gamma(\alpha)$ 是 gamma 函数。最佳的 $\alpha 、 \beta$ 估计值可采用极 大似然估计方法求得，即

$$
\begin{aligned}
& \hat{\alpha}=\frac{1+\sqrt{1+4 A / 3}}{4 A} \\
& \hat{\beta}=\frac{\bar{x}}{\hat{\alpha}} \\
& A=\ln (\bar{x})-\frac{\sum \ln (x)}{n}
\end{aligned}
$$

式中 , $n$ 为计算序列的长度。于是 给定时间尺度的 累积概率可计算如下： 


$$
\begin{aligned}
& G(x)=\int_{0}^{x} g(x) d x=\frac{1}{\hat{\beta}^{\alpha} \Gamma(\hat{\alpha})} \int_{0}^{x} x^{\alpha-1} e^{-x / \beta} d x \\
& \text { 令 } t=x / \hat{\beta}
\end{aligned}
$$

上式可变为不完全的 gamma 方程 :

$$
G(x)=\frac{1}{\Gamma(\hat{\alpha})} \int_{0}^{x} t^{\hat{a}-1} e^{-t} d t
$$

由于 gamma 方程不包含 $x=0$ 的情况, 而实际 降水量可以为 0 , 所以累积概率表示为:

\section{$H(x)=q+(1-q) G(x)$ 。}

式中, $q$ 是降水量为 0 的概率。如果 $m$ 表示降 水时间序列中降水量为 0 的数量, 则 $q=m / n$ 。

累积概率 $H(x)$ 可以通过下式转换为标准正态 分布函数。

当 $0<H(x) \leqslant 0.5$ 时:

$$
\begin{gathered}
Z=S P I=-\left(t-\frac{c_{0}+c_{1} t+c_{2} t^{2}}{1+d_{1} t+d_{2} t^{2}+d_{3} t^{3}}\right) \\
t=\sqrt{\ln \left[\frac{1}{H(x)^{2}}\right]}
\end{gathered}
$$

当 $0.5<H(x)<1$ 时：

$$
\begin{aligned}
Z & =S P I=\left(t-\frac{c_{0}+c_{1} t+c_{2} t^{2}}{1+d_{1} t+d_{2} t^{2}+d_{3} t^{3}}\right) \\
t & =\sqrt{\ln \left\{\frac{1}{[1.0-H(x)]^{2}}\right\}} \\
c_{0} & =2.515517 ; c_{1}=0.802853 ; c_{2}=0.010328 ; \\
d_{1} & =1.432788 ; d_{2}=0.189269 ; d_{3}=0.001308
\end{aligned}
$$

据此, 可以求得 $S P I$, 其旱涝等级值见表 1 。

$Z$ 指数 :由于某一时段的降水量一般并不服从 正态分布, 为此用 Person III 型分布拟合某一时段的 降水量效果较好( 幺枕生等, 1990)。 $Z$ 指数正是假 设某时段的降水量服从 Person III 型分布, 其概率密 度函数为：

$$
f(x)=\frac{\beta}{\Gamma_{(\alpha)}}(x-\alpha)^{\alpha-1} e^{-\beta(x-\alpha)},(x>\alpha)
$$

而后对降水量进行正态化处理, 这样可将概率 密度函数 Person III型分布转换为以 $Z$ 为变量的标准 正态分布。转换公式为：

$$
Z_{i}=\frac{6}{C_{s}}\left|\frac{C_{s}}{2} \Phi_{i}+1\right|^{\frac{1}{3}}-\frac{6}{C_{s}}+\frac{C_{s}}{6}
$$

式中: $C_{s}$ 为偏态系数, $\Phi_{i}$ 为标准变量, 计算公式分 别为:

$$
C_{s}=\frac{\sum_{i=1}^{n}\left(X_{i}-\bar{X}\right)^{3}}{n \sigma^{3}}, \Phi_{i}=\frac{X_{i}-\bar{X}}{\sigma}
$$

式中：

$$
\sigma=\sqrt{\frac{1}{n} \sum_{i=1}^{n}\left(X_{i}-\bar{X}\right)^{2}}, \bar{X}=\frac{1}{n} \sum_{i=1}^{n} X_{i}
$$

根据 $Z$ 变量的正态分布曲线，划分为 7 个等 级, 并确定其相应的 $Z$ 界限值, 各级旱涝指标 见表 1 。

\section{2 研究资料}

为比较 $S P I$ 和 $Z$ 指数,且使之具有代表性,在 全国不同气候区选择 7 个代表气象站(北京、成都、 济南、昆明、兰州、锡林浩特、武汉) 的 1951 1995 年 气象资料。除锡林浩特气象站为 43 年 (1953 1995 年)资料外，其余 6 站均为 45 年。

\section{2 结果与分析}

\section{$2.1 S P I$ 和 $Z$ 指数的单站一致性比较}

对全国 7 个代表性气象台站 45 年间(锡林浩特 为 43 年) 每月的 $S P I$ 和 $Z$ 指数的计算后发现, SPI 和 $Z$ 指数在衡量旱涝程度上具有很好的一致性, 并 利用 SPSS 统计软件进行 Mann-Whitney 方法的一致 性检验。所得结果见表 2。各站所得 $p$ 值均大于 $0.05, S P I$ 和 $Z$ 指数在衡量单站单月旱涝程度上具 有极显著的一致性。图 1 给出了 1986 1995 年昆 明和锡林浩特每月的 SPI 和 $Z$ 指数等级值。

\section{$2.2 S P I$ 和 $Z$ 指数对比分析}

$S P I$ 和 $Z$ 指数在计算时是把相同月份的降水量 作为一个序列来衡量, 某月的旱涝程度是与长时间 序列的平均水平相比较做出的, 某月的降水量较大 则对应的指标值也较大 (趋向于湿润), 反之对应的 指标值也较小 (趋向于干旱)。所以, 能否准确反映

表 $1 S P I$ 和 $Z$ 指数的旱涝等级

Table 1 Classification scales for $S P I$ and $Z$-index

\begin{tabular}{cccc}
\hline 等级 Scale & 标准化降水指标 $S P I$ & $Z$ 值 $Z$-index & 类型 Moisture category \\
\hline 1 & $>2$ & $>1.96$ & 重涝 Extreme wet \\
2 & $(1.5,2)$ & $(1.44,1.96)$ & 中涝 Moderate wet \\
3 & $(1,1.5)$ & $(0.84,1.44)$ & 轻涝 Slight wet \\
4 & $(-1,1)$ & $(-0.84,0.84)$ & 正常 Normal \\
5 & $(-1.5,-1)$ & $(-1.44,-0.84)$ & 轻旱 Slight drought \\
6 & $(-2,-1.5)$ & $(-1.96,-1.44)$ & 中旱 Moderate drought \\
7 & $<-2.00$ & $<-1.96$ & 重旱 Extreme drought \\
\hline
\end{tabular}




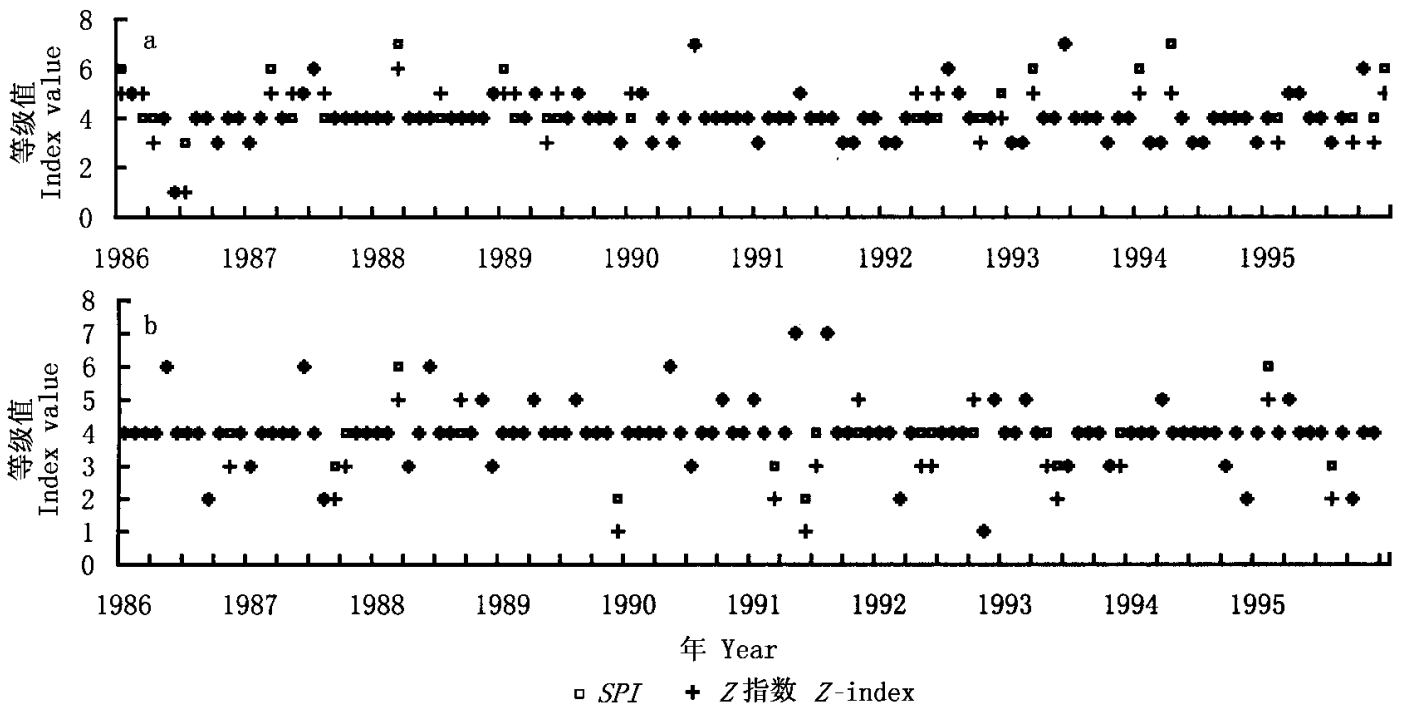

图 $1 S P I$ 和 $Z$ 指数等级值比较

Fig. 1 Class values of $S P I$ and $Z$-index $\mathrm{a}$ : 昆明 Kunming b : 锡林浩特 Xilinhaote

表 2 各站一致性

Table 2 Coherence of 7 stations

\begin{tabular}{lc}
\hline 站点 Station & $p$ \\
\hline 北京 Beijing & 0.697 \\
成都 Chengdu & 0.976 \\
济南 Jinan & 0.955 \\
昆明 Kunming & 0.997 \\
兰州 Lanzhou & 0.822 \\
锡林浩特 Xilinhaote & 0.682 \\
武汉 Wuhan & 0.692 \\
\hline
\end{tabular}

旱涝变化,能否遵循降水量大小变化趋势是评价 $S P I$ 和 $Z$ 指数优劣的重要标准。虽然两者在全国不 同气候区域评价旱涝程度时取得极显著的一致性， 但是由于两种指标在计算过程中采取不同的技术路 线, 因而对于旱涝程度的衡量效果不尽相同。研究 发现, SPI 的大小变化趋势在所考察的 7 个站点中 的各个月份均与降水量有着极好的对应关系，对旱 涝变化反映敏感, 能够有效地衡量各个时段的旱涝 状况。而 $Z$ 指数值与降水时空分布特性有着密切 的联系，在不同地区和不同时段对旱涝的敏感性不 尽相同, 在某些时段 $Z$ 指数值大小的变化趋势与降 水量的趋势并不一致，难以有效地反映旱涝程度。

如表 3 所示，以北京 10 月的降水量从小到大排 序, SPI 值极好地遵循同样的规律, $Z$ 指数则出现了 异常的序列值。在降水量 0 到 $5.4 \mathrm{~mm}$ 的变化区间 内, 随着降水量的增大, $Z$ 指数反而呈现出递减的趋 势, 直到从 $5.5 \mathrm{~mm}$ 开始 $Z$ 指数才恢复正常的递增 趋势。但是即使如此 1957 年和 1962 年降水量为 0 ,
其所对应的 $Z$ 指数值却为 -0.04 , 最大超过了降水 量为 $10.3 \mathrm{~mm}$ 的 $Z$ 指数值, 这种现象与正常的 $Z$ 指 数理论相矛盾。

为找出 $Z$ 指数异常值的出现规律, 进行如下的 理论分析。假设在长时间序列的某月降水量值中有 $X_{1} 、 X_{2}$ ，且 $X_{1}>X_{2}$ 异常时 $Z_{1}<Z_{2}$ 则有:

$$
\left|\frac{\frac{C_{s}}{2} \Phi_{1}+1}{\frac{C_{s}}{2} \Phi_{2}+1}\right|<1
$$

求解不等式可得 $Z$ 指数出现异常的条件：

$$
X_{1}+X_{2}<\frac{-4 \sigma}{C_{s}}+2 \bar{X}
$$

随着右式的增大，出现 $Z$ 指数计算异常的可能 性也会相应的变大。 $Z$ 指数值计算是基于降水量, 但是其所表现出的趋势却在这时与降水量的变化趋 势相反。这说明 $Z$ 指数计算公式存在较为严重的 漏洞，在极端情况下表现出了上述的异常结果。相 比而言, SPI 是根据降水量的概率分布计算累积概 率 然后再转化成标准正态分布而得, 与 $Z$ 指数相 比具有优良的计算稳定的特性，很好地符合实际降 水的变化趋势, 明显地反映了旱涝程度。

\section{$2.3 S P I$ 多时间尺度应用特性的例证}

由于干旱自身的复杂性和对社会影响的广泛 性, 在评价干旱程度及其影响时需要了解不同时间 尺度上的水分变化。目前, 不同部门、不同地区根据 各自的应用需求，使用不同时间尺度的干旱指标，这 样大大降低了干旱程度之间的可比性和对干旱监测 
表 3 北京部分年份 10 月的降水量、 $Z$ 指数和 $S P I$ 值 Table 3 Precipitation、 $Z$-index and SPI in October partial years in Beijing

\begin{tabular}{cccc}
\hline 年份 & $\begin{array}{c}\text { 降水量 } \\
\text { Year }\end{array}$ & $\begin{array}{c}Z \text { 指数 } \\
\text { Precipitation }(\mathrm{mm})\end{array}$ & $\begin{array}{c}\text { 标准化降水 } \\
\text { 指标 } S P I\end{array}$ \\
\hline 1957 & 0.0 & -0.04 & -1.70 \\
1962 & 0.0 & -0.04 & -1.70 \\
1990 & 0.3 & -0.07 & -1.64 \\
1975 & 1.6 & -0.19 & -1.33 \\
1965 & 3.5 & -0.45 & -1.00 \\
1985 & 3.8 & -0.51 & -0.96 \\
1986 & 4.1 & -0.57 & -0.92 \\
1987 & 4.1 & -0.57 & -0.92 \\
1989 & 4.6 & -0.70 & -0.85 \\
1979 & 4.7 & -0.73 & -0.84 \\
1963 & 5.4 & -1.24 & -0.76 \\
1971 & 5.5 & -1.11 & -0.74 \\
1952 & 5.7 & -0.93 & -0.72 \\
1969 & 5.7 & -0.93 & -0.72 \\
1982 & 8.5 & -0.28 & -0.44 \\
1995 & 9.6 & -0.16 & -0.35 \\
1993 & 9.9 & -0.13 & -0.33 \\
1961 & 10.0 & -0.12 & -0.32 \\
1974 & 10.0 & -0.12 & -0.32 \\
1994 & 10.3 & -0.09 & -0.29 \\
\hline
\end{tabular}

的一致性。SPI 通过求算给定时间尺度的累积概 率, 使其能够在多个时间尺度上进行计算。从而 SPI 不仅可以用于监测短时期内的水分变化, 如对 农业生产有着重要影响的土壤水分变化, 而且还可 以监测长时期的水分动态, 如地下水供给、径流量 等。虽然, $Z$ 指数在反映单月旱涝的同时, 也可以反 映不同时间尺度的水分变化。但是, 如前所述, $Z$ 指 数在计算上的不稳定性同样会影响多时间尺度指标 值的计算结果。

本文以北京 $1951 \sim 1995$ 年 4 个时间尺度的 SPI 指标值为例说明其多时间尺度的使用特性。

北京在 $1951 \sim 1995$ 年期间发生了 3 次严重的 干旱(六振达,1996)。1965 年北京降水量显著减少, 年降水量仅 $377 \mathrm{~mm}$,出现了历史上严重的干旱，引 起了城市的供水危机; 1970 1972 年 3 年连旱, 年均 降水量仅为 $508 \mathrm{~mm}$, 造成 200 多万亩作物减产, 最 后依靠大量打井超采地下水得以度过; 20 世纪 80 年代初连续 5 年干旱, 水库水位大幅度下降, 农业受 灾面积达 300 多万亩。在这 45 年期间发生过最大 规模的洪涝灾害是在 1959 年。1959 年 7 月下旬至 8 月下旬降水量比同期增长 $70 \%$, 累积平均降水量达 到 $1051.8 \mathrm{~mm}$ 致使农田大面积受灾, 城区进水(李 裕宏, 1999)。

通过计算 12 个月和 24 个月时间尺度的 SPI 值
(分别标记为 $S P I_{12}$ 和 $S P I_{24}$ ), 可以明确地标示出 3 次旱灾和 1959 年的涝灾 (图 2c、d)。 $S P I_{12}$ 与 $S P I_{24}$ 的 柱状图相比有较大的正负波动。不同时间尺度的 SPI 值对于降水量的敏感性不同，时间尺度越小，则 对于一次降水变化越显著, 其值会发生较大变化, 甚 至是正负波动。相反，时间尺度越大则对于一次降 水的反映并不显著，只有持续的多次降水才会使之 发生波动。这对于监测长时期的水分状况是合理的 (Seiler et al. 2002)。SPI 12 和 $S P I_{24}$ 都显示出虽然在 1957 年出现了短暂的轻微水分亏缺, 但是总体而 言，从 1954 年起北京的水分充足, 说明下层土壤水 分、径流量、地下水以及水库蓄水量丰富。因而， 1959 年 7、8 月间的大量降水不能有效地经由土壤下 渗、地表径流和水库蓄水分担，造成了北京 45 年间 最严重的涝灾。长期的水分富足状态 $\left(S P I_{12}\right.$ 和 $S P I_{24}$ 保持较大的正值) 和短时间大量的降水 $\left(S P I_{1}\right)$ 可以很好地成为指示 1959 年涝灾的信号。

$S P I_{12}$ 和 $S P I_{24}$ 在 $1965 \sim 1969$ 年、1970 1972 年 和 1980 1985 年 3 个时期内持续保持较大的负值, 这与历史记录中北京 3 次大规模的干旱有极好的对 应关系。3 个时期内的 $S P I_{1}$ (单月的 $S P I$ 值)和 $S P I_{3}$ (3 个月的 $S P I$ 值)也有着相应的变化趋势。如 $S P I_{3}$ 在 1965 年 7 月、1968 年 3 月和 1975 年 4 月等前后出 现了较为明显的水分亏缺。 $S P I_{1}$ 也有着类似的趋 势, 只是期间的波动更为明显。这是因为 $S P I_{1}$ 对于 每次降水变化都极为显著。由此可见, 应用多时间 尺度的 SPI 指标值配合分析, 能够清楚地得到不同 方面的水资源状况。 $S P I_{1}$ 可以监测每月的水分变 化，可以准确地反映土壤水分状况，这对于适时作好 排水灌溉, 保证农业生产是极为必要的。 $S P I_{12}$ 和 $S P I_{24}$ 对于下层土壤水分、河流径流量、地下水位以 及水库蓄水量有较好地反映。综合分析多时间尺度 的 SPI 值可以有效地反映旱涝程度及其持续时间。

\section{3 结 论}

干旱指标的发展经历了由最初只是反映降水多 少的简单指标到现在建立针对某一具体问题的复杂 模型(如 PDSI、KBDI)。我国科学工作者在干旱指标 的制定和应用方面作了大量工作，但是由于干旱的 复杂性和影响的广泛性, 迄今为止还没有建立起完 善统一的干旱指标体系，不同地区、不同领域使用不 同尺度的干旱指标, 这样大大降低了干旱等级的可 比性和干旱监测的连续性。SPI可以计算不同时间 


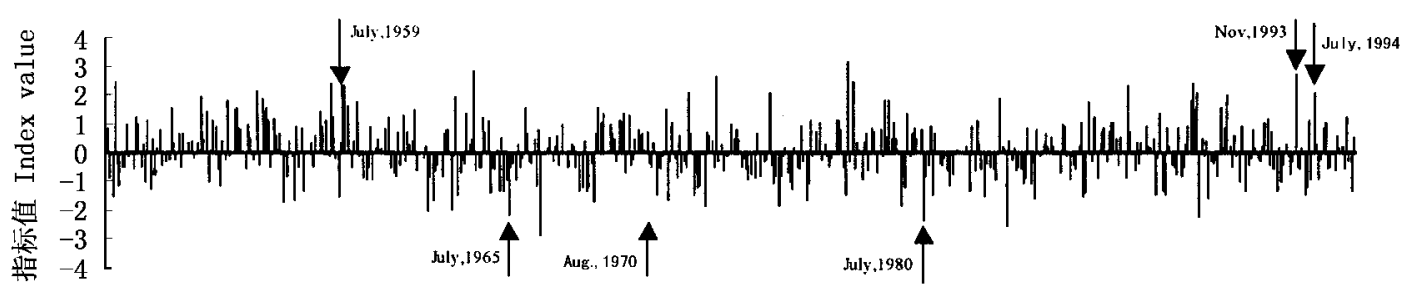

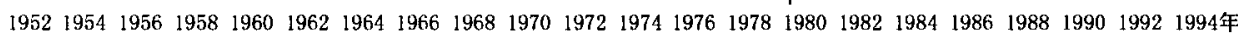

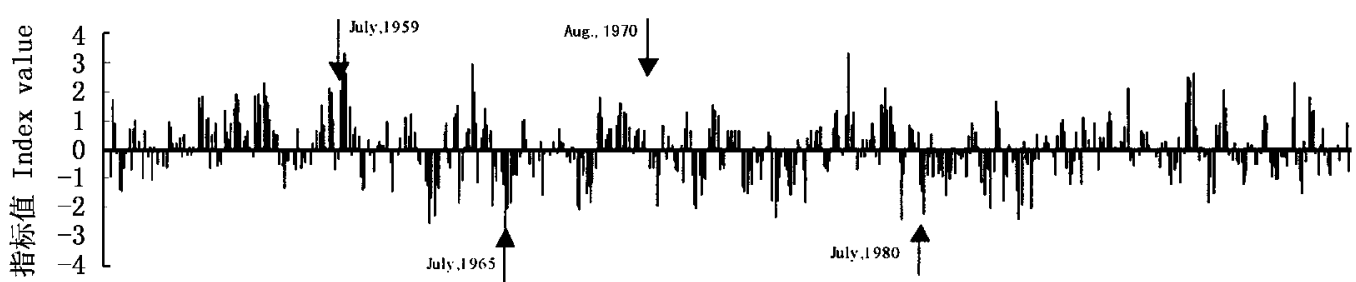

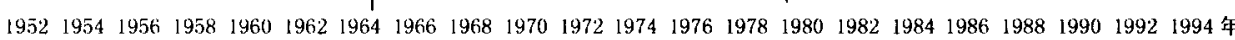

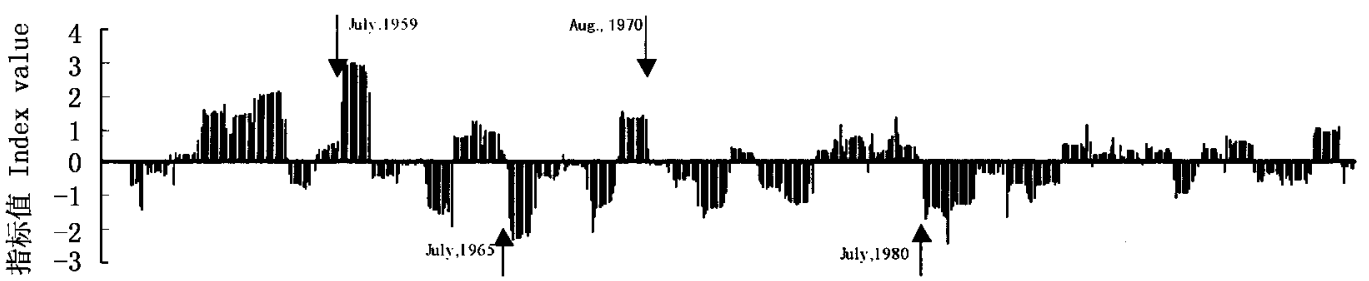

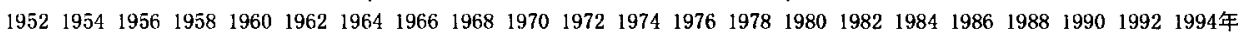

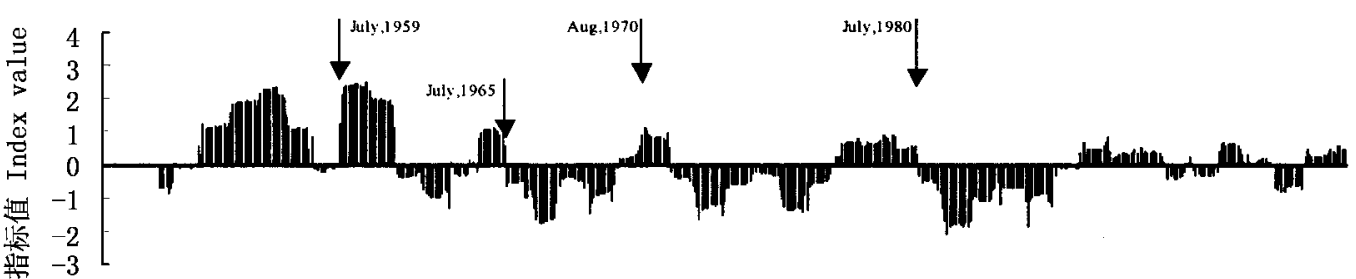

$19521954 \quad 19561958 \quad 196019621964 \quad 1966 \quad 1968 \quad 19701972 \quad 1974 \quad 1976 \quad 1978 \quad 19801982 \quad 1984 \quad 1986 \quad 1988 \quad 19901992 \quad 1994 \quad$ 年

图 $21951 \sim 1995$ 年北京 SPI 多时间尺度分析

Fig.2 SPI values calculated form the $1951-1995$ series for different periods in Beijing

$\mathrm{a}$ : 单月 1 month $\left(S P I_{1}\right) \quad \mathrm{b}: 3$ 个月 3 months $\left(S P I_{3}\right) \quad$ c : 12 个月 12 months $\left(S P I_{12}\right) \quad$ d : 24 个月 24 months $\left(S P I_{24}\right)$

尺度的指标值, 满足多种水分监测需求, 可以为不同 部门和领域提供一个统一的干旱指标。

通过对 SPI 与 $Z$ 指数一致性比较和多时间尺 度分析, 得到如下结论 :

1) $S P I$ 和 $Z$ 指数的计算只需要降水作为输入 量, 资料容易获取, 也避免了机理模型繁杂的计算和 大量经验性的参数输入, 同时又抓住了反映干旱的 主项 降水。而且由于不涉及具体的干旱机理, 其时 空适应性强, 克服了机理性干旱指标因为寒冷、地 形、土壤类型等因素所造成的使用上的制约。

2) $S P I$ 和 $Z$ 指数两者用非正态分布拟合某一时 段的降水量，再经过正态化处理，在计算原理上具有 一定的相似性，这也是两者在全国不同气候区域评 价旱涝等级时得到一致性极显著的原因，也显示了 在一定程度上两者的可替代性。但是两种指标在计 算过程中采取不同的技术路线，因而对于旱涝程度
的衡量效果不尽相同。 $Z$ 指数是把概率密度函数直 接标准化, 其中涉及到偏态系数、标准变量等参数, 表明 $Z$ 指数的大小不仅与降水量有关, 还与降水的 时空分布有关。鞠笑生等 (1997) 研究指出, $Z$ 指数 分析效果与偏态系数密切相关, 偏态系数越大, $Z$ 指 数的分析结果越好, 越能反映出旱涝的程度。因而 不同地区或不同月季降水的差异会导致 $Z$ 指数使 用效果的差异。SPI 则是通过概率密度函数求解累 积概率，再将累积概率标准化，计算过程中没有涉及 与降水量的时空分布特性有关的参数, 降低了指标 值计算的时空变异，对不同时空的旱涝状况都有良 好的反映。通过对 SPI 和 $Z$ 指数对比分析后发现， 与 $Z$ 指数相比, SPI 具有更加优越的计算稳定的特 性。

3) $S P I$ 同 $Z$ 指数一样存在因不涉及干旱机理而 产生的不足。首先, 由于 $S P I$ 的计算特性, 不同地点 
的干旱等级频度相同，即假定了所有地点发生旱涝 极端事件的概率相同, 无法标识旱涝频发地区。其 次, 除由于降水偏少影响以外, 气候变暖蒸发加大也 是造成干旱的重要因素(方修琦等, 1997) ,而 SPI 没 有考虑气温、蒸发对干旱的影响。最后, SPI 值的计 算是建立在长时间序列基础上的,其单月值是在该 时间序列同一时期平均水平上的反映。与湿季同样 多的甚至是少的降水量在旱季的 SPI 值会大得多。 例如, 在图 2a中 1993 年 11 月的降水量只有 43.4 $\mathrm{mm}$, 远低于 1994 年 7 月 $459.2 \mathrm{~mm}$ 的降水量, 但是其 $S P I$ 值却为 $2.74,1994$ 年 7 月的指标值为 2.05 。其 原因在于北京地区 11 月份的平均降水量仅为 7.7 $\mathrm{mm} 43.4 \mathrm{~mm}$ 的降水量就会得到较大的 SPI 值。但 是这并不意味着 1993 年 11 月发生了重涝事件。这 是由于 SPI 值是建立在时间序列中同期水平的基础 上, 具有一定的合理性。另一方面也说明在利用 $S P I$ 进行旱涝分析时必须结合具体情况加以分析。

4) 由于 SPI 多时间尺度的特性, 从而使得用同 一个干旱指标反映不同时间尺度和不同方面的水资 源状况成为可能。而且, SPI 可以直接区分引起干 旱的两种直接原因: 土壤水分亏缺和用于补给的水 分亏缺(Byun \& Wilhite, 1999)。土壤水分亏缺是由 于目前的降水减少而致，而用于补给的水分亏缺则 与长期的降水缺乏有关。考虑两种水分亏缺过程并 探讨两者的关系, 可以提高干旱的预测精度和及时 性。

SPI 计算简单易行, 资料容易获取, 同时在各个 地区和各个时段都具有良好的计算稳定性, 能有效 地反映旱涝状况，优于在我国有着成熟应用的 $Z$ 指 数。而且还具有优越的多时间尺度应用特性, 可以 满足不同地区、不同应用的需求，因而可以为我国的 水资源评估和不同时间尺度的干旱监测服务。

\section{参 考 文 献}

Byun, H. R. \& D. A. Wilhite. 1999. Objective quantification of drought severity and duration. Journal of Climate, 12: 2747 2756 .

Fang, X. Q. (方修琦) , Y. R. He(何英茹) \& W. B. Zhang(章 文波). 1997. An EOF analysis on drought effect on agriculture in China during 1978 1994. Journal of Natural Disasters(自然 灾害学报),6(1):59 64. (in Chinese with English abstract)

Felix, N. K. 1997. Global drought watch from space. Bulletin of American Meteorological Society, 78:621 636.

Hayes, M. J., M. D. Svoboda, D. A. Wilhite \& O. V. Vanyarkho. 1999. Monitoring the 1996 drought using the standardized precipitation index. Bulletin of the American Meteorological Society, 80:429 438.

Ju, X. S. (鞠笑生) , X. W. Yang (杨贤为), L. J. Chen(陈丽 娟) \& Y. M. Wang(王有民). 1997. Research on determination of station indexes and division of regional flood/drought grades in China. Quarterly Journal of Applied Meteorology (应用气象学 报), 8:26 33. (in Chinese with English abstract)

Ju, X. S. (鞠笑生), X. K. Zou (邹旭恺) \& Q. Zhang (张强). 1998. The method of climatic drought-flood index and its analysis. Journal of Natural Disasters(自然灾害学报), 7(3):51 57. (in Chinese with English abstract)

Li, Y. H. (李裕宏). 1999. Review on flood of 1959 in Beijing. Beijing Water Resources (北京水利), (4):21 24. (in Chinese)

Liu, Z. D(六振达). 1996. Review and consider on water crisis in Beijing. Beijing Water Resources (北京水利), (6):8 9. (in Chinese)

Palmer, W. C. 1965. Meteorological drought . U.S. Weather Bureau Research Paper. $45 \sim 58$.

Richard, R. \& J. R. Heim. 2002. A review of twentieth-century drought index used in the United States. Bulletin of American Meteorological Society, 83: $1149 \sim 1165$.

Seiler, B. A. , M . Hayes \& L. Bressan. 2002. Using the standardized precipitation index for flood risk monitoring. International Journal of Climatology, 22:1365 1376 .

Wang, Y.L. (王延禄).1990. The summa concerning arid meteorological targets to establish, quote and test in China. Arid Land Geography (干旱区地理), 13 (3): 80 86. (in Chinese with English abstract)

Wilhite, D. A. 2000. Drought as a natural hazard: concepts and definitions. In: Wilhite, D. A. ed. Drought: a global assessment. New York: Routledge. 3 18.

Yao, Z. S. (枕生) \& Y. G. Ding (丁裕国). 1990. Climate statistics. Beijing: China Meteorological Press. $161 \sim 180$. (in Chinese)

Zierl, B. 2001. A water balance model to simulate drought in forested ecosystems and its application to the entire forested area in Switzerland. Journal of Hydrology, 242:115 136 .

责任编委 : 刘世荣 责任编辑 :姜联合 